\title{
Tradução e adaptação cultural do Index of Premature E jaculation (IPE) para o português do Brasil
}

\author{
Ítor Finotelli Júnior- UnivesidadeSão Franciso Itatiba, São Paulo Brasil \\ CláudioGaria Capitão- UnivesidadeSão Franişo, Itatiba, SãoPaulo, Brasil
}

\begin{abstract}
Resumo
O presente estudo traduziu e adaptou o Index of PrematureEjaalation(IPE) para o português do Brasil, por método de equivalência semântica. O IPE é uma escala em autorrelato composta por 10 itens que avaliam critérios como senso de controle, satisfação sexual e sofrimento. Compuseram esse processo 11 profissionais qualificados para tal método e uma amostra de 50 participantes para avaliação quanto à inteligibilidade. As etapas foram: tradução, retrotradução, avaliação da equivalência semântica, crítica final por especialistas e pré-teste da versão. Os resultados para avaliação da equivalência apresentaram concordância significativa entre os juízes. Essa avaliação classificou os itens como inalterados ou pouco alterados. A crítica final por especialista avaliou qualitativamente as divergências e consolidou a versão aplicada na amostra. Após essa aplicação, algumas sugestões foram incorporadas e constituíram a versão final. Os resultados foram satisfatórios na compreensão da linguagem empregada. Para essa versão, conservou-se o nome em inglês, acrescido ao final de "adaptação brasileira".

Palavasdhave Ejaculação precoce; Disfunção sexual; Escala; Equivalência semântica.
\end{abstract}

\section{Translation and cultural adaptation of the Index of Premature Ejaculation (IPE) to Brazilian Portuguese}

\begin{abstract}
The present study translated and adapted the Index of PrenatureEjaalation(IPE) into Brazilian Portuguese, by the method of semantic equivalence. The IPE is a self-report scale composed by 10 items that assesses criteria such as sense of control, sexual satisfaction and distress. This process was composed by 11 professionals qualified for such method and a sample of 50 participants for the evaluation of intelligibility. The stages were translation, back-translation, assessment of semantic equivalence, final critique by experts and version pre-test. The results for the assessment of semantic equivalence presented significant agreement among the judges. This assessment classified the items as unchanged or slightly changed. The final critique by experts evaluated qualitatively the divergences and consolidated the version applied in the sample. After this application, some suggestions were incorporated and these constituted the final version. The results were satisfactory in the comprehension of the adopted language. For this version, the name was kept in English and "Brazilian Adaptation" was added to the end.
\end{abstract}

Kegnards Premature ejaculation; Sexual dysfunction; Scale; Semantic equivalence.

\section{Traducción y adaptación cultural del Index of Premature E jaculation (IPE) al portugués de Brasil}

\begin{abstract}
Resumen
El presente estudio tradujo y adaptó el Indax of Premature Ejaalation(IPE) para el portugués de Brasil, por método de equivalencia semántica. El IPE es una escala de autoinforme compuesta por 10 ítems que evalúan criterios como sentido de control, satisfacción sexual y sufrimiento. Participaron de ese proceso 11 profesionales calificados para tal método y una muestra de 50 participantes para evaluación con referencia a la inteligibilidad. Las etapas fueron traducción, retrotraducción, evaluación de la equivalencia semántica, crítica final por expertos y pretest de la versión. Los resultados para la evaluación de la equivalencia presentaron concordancia significativa entre los jueces. Esa evaluación clasificó los ítems como inalterados o poco alterados. La crítica final por expertos evaluó cualitativamente las divergencias y consolidó la versión aplicada en la muestra. Después de esa aplicación, algunas sugerencias fueron incorporadas y se constituyó la versión final. Los resultados fueron satisfactorios en la comprensión del lenguaje empleado. Para esa versión se conservó el nombre en inglés, añadiéndose al final "adaptación brasileña".

Palabrasdave Eyaculación precoz; Disfunción sexual; Escala; Equivalencia semántica.
\end{abstract}

Existe uma variação substancial entre os homens ao longo do contínuo de latência da ejaculação. Em sua maioria, os relatos desse período variam de 2 a 10 minutos. No entanto, alguns homens ejaculam incontrolavelmente antes ou logo após uma penetração e, consequentemente, podem ser classificados como tendo uma ejaculação precoce (EP). No caso oposto, existem também homens com dificuldade em alcançar a ejaculação, sendo eles classificados com retardo ou inibição ejaculatória. Esses homens não atingem a ejaculação e/ ou o fazem após a estimulação prolongada (Rowland, Tai \& Brummett, 2007).
A ejaculação é a resposta do organismo que permite a emissão e expulsão do sêmen mediante a estimulação (Kedia, 1983). Um "reflexo que compreende áreas e receptores sensoriais, vias aferentes, áreas sensoriais cerebrais, centros motores cerebrais, vias eferentes e centros motores espinais" (Ankier \& Glina, 2005, p. 8), predominantemente "controlada pela interação complexa entre os neurônios serotoninérgicos e dopaminérgicos centrais com 0 envolvimento secundário dos neurônios colinérgicos, adrenérgicos, oxitocinérgicos e GABAnérgicos" (Ankier \& Glina, 2005, p. 8). 
Por ser modulada por processos centrais, não surpreende 0 relato de homens no controle sobre sua temporização (Rowland, Strassberg, de G ouveia Brazao \& Slob, 2000). Em contrapartida, por se tratar de uma resposta regular, nenhuma falha ou incapacidade pode ser justificada por aspectos fisiológicos, caso ela ocorra tão rapidamente (Levin, 2005).

Referente à problemática, os estudos sobre a EP são dificultados pela falta de consenso sobre que critérios a definem como uma disfunção, doença ou condição (Segraves, 2010). Observa-se que a mesma dificuldade é encontrada para sua nomenclatura, cujas denominações variam: ejaculação rápida, precoce, prematura, involuntária, não-controlada, fraca, entre outras (Althof, 2004).

A justificativa para a EP ser considerada um problema possivelmente emergiu da conscientização em torno do prazer sexual feminino. Nessa abordagem, pesquisadores como Masters e Johnson (1970) definiram a EP como a impossibilidade do retardo do reflexo ejaculatório por tempo suficiente, durante 0 intercurso, para satisfazer uma parceria receptiva em $50 \%$ das experiências coitais. Embora esse critério pareça útil e recomendado como complemento nas investigações clínicas em parcerias sexuais (Rodrigues Jr., 1995), a variabilidade da rapidez e a capacidade entre as mulheres limitam sua utilidade.

Nessa direção, avaliar um indivíduo com uma disfunção sexual incluirá outros critérios, além da função sexual. Trata-se de uma condição influenciada por múltiplas variáveis (Leiblum, 2007). Por essa razão, compreender as expectativas desse indivíduo a respeito das atividades sexuais e afetivas cria direções para critérios mensuráveis entre as inúmeras formas de prazeres sexuais, além da penetração (Althof, 2007).

Para compreensão da EP, algumas propostas para critérios objetivos sugiram na literatura. 0 tempo ejaculatório foi uma delas (Waldinger, Hengeveld, Zwinderman \& Olivier, 1998). Trata-se da avaliação por meio do tempo de latência intravaginal para a ejaculação (IELT), definido pelo número em segundos/ minutos entre a inserção do pênis na vagina até a ejaculação, calculado a partir da média sobre um número de tentativas (Waldinger, Q uinn \& cols., 2005; Waldinger, Zwinderman, Olivier \& Schweitzer, 2005). Segundo Waldinger (2007), essa é a mensuração mais objetiva sobre a EP. Apesar de útil para triagens, as pesquisas ainda não estabeleceram um consenso sobre sua estimativa.

Para além do critério tempo, destacaram-se na literatura outros critérios mensuráveis, como o senso de controle e a satisfação sexual. Segundo Rowland (2003), estes ganharam importância, ao considerar a multidimensionalidade de fatores relacionados à EP.
Pesquisas relacionadas ao senso de controle demonstraram que homens que não indicaram problemas de ejaculação relataram um controle bastante elevado sobre 0 momento da ejaculação em comparação a homens com tempos curtos de latência ejaculatória (Byers \& Grenier, 2003; Rowland, Cooper \& Schneider, 2001; Rowland \& cols., 2000). Para satisfação sexual, a insatisfação é relatada por homens e mulheres na existência de uma EP (Davies, Katz \& Jackson, 1999; Larson, Anderson, Holman \& Niemann, 1998; Rust, Golombok \& Collier, 1988).

Adicionalmente a esses dois critérios, 0 sofrimento também foi relacionado à EP e, em razão de sua importância, foi incluído como critério na definição mais recente e amplamente utilizada entre as estabelecidas. Essa definição foi formulada no $2^{\underline{0}}$ Consenso Internacional de Medicina Sexual e estabeleceu a EP como a ejaculação que ocorre antes do desejado, tanto antes como logo após a penetração, e sobre a qual o homem tem mínimo ou nenhum controle. Essa ausência de controle é acompanhada por elevado grau de sofrimento ou dificuldade interpessoal, incômodo, frustração e/ ou evitação da atividade sexual (Lewis \& cols., 2004).

A EP é considerada uma situação embaraçosa que ocorre em práticas sexuais, como carícias, beijos, sexo oral e anal, com ou sem roupas (Jern, 2010; Leiblum, 2007). Apesar da dificuldade no estabelecimento de critérios e nomenclatura permanentes em comparação às outras disfunções sexuais, ela é uma das disfunções masculinas mais comuns (Porst \& cols., 2010).

Para os instrumentos disponíveis utilizados na sua avaliação, quatro foram encontrados na literatura, 0 Premature Ejaallation Questionnaire - PEQ uest (Hartmann, Schedlowski \& Kruger, 2005), o Chimese Index of Premature Ejaalation - CIPE (Yuan \& cols., 2004), o Premature Ejaallation Profile - PEP (Patrick \& cols., 2009) e 0 Index of Premature Ejaalation - IPE (Althof \& cols., 2006). De maneira geral, são instrumentos breves, que variam de 10 a 36 itens por autorrelato, de fácil administração em níveis de medidas diferenciados. Por outro lado, a verificação dos estudos de validade e das qualidades psicométricos entre os citados indicaram uma situação preocupante.

Apenas o PEP e o IPE apresentaram em seus estudos mais de uma evidência de validade, sendo 0 PEP um instrumento de rastreamento contendo quatro itens. O PEQuest não apresentou ou justificou a ausência de informações estatísticas referentes ao processo de validação, assim como o CIPE para versão traduzida para 0 inglês. Para esses dois últimos, os autores apresentaram um número de dimensões avaliadas da EP incompatíveis com a quantidade de itens. Em média, dois itens para cada dimensão. Por 
fim, todos possuem itens e/ ou dimensões que avaliam os critérios mencionados anteriormente, 0 tempo ejaculatório, o senso de controle, a satisfação e 0 sofrimento.

Nenhum deles possui tradução, adaptação e/ ou validade para 0 português do Brasil. Para os instrumentos citados, a revisão em publicações dos últimos 20 anos foi feita por Porst e colaboradores (2010), afirmaram que o IPE é aquele que melhor ilustra o exercício de criação e validação de um instrumento, ao considerar etapas criteriosas nesse processo.

O IPE é uma escala de autorrelato com 10 itens que avaliam a EP por critérios como tempo ejaculatório, senso de controle, satisfação sexual e sofrimento. Quatro etapas foram utilizadas no processo de construção. Na primeira, foi criado um banco de 17 itens por dois especialistas. Na etapa seguinte, uma aplicação desses itens foi realizada para avaliação das propriedades psicométricas. Com os dados dessa aplicação, os pesquisadores empregaram uma análise fatorial exploratória por componentes principais com rotação varimax. Os critérios utilizados para extração dos fatores foram 0 autovalor $>1,0 \mathrm{e}$ retenção de itens com cargas iguais ou superiores a 0,4 (Althof \& cols., 2006).

Os resultados indicaram quatro dimensões para extração, todavia foram identificados itens que não contribuíram estatisticamente para sua composição. Procedeu-se à exclusão desses itens e uma nova análise fatorial foi efetuada. Ela extraiu três dimensões que cumpriram os critérios expostos, nomeadas de controle ejaculatório, satisfação sexual e sofrimento. Observaram-se associações significativas entres as dimensões. A precisão delas foi estimada pela consistência interna e estabilidade temporal; a primeira variou entre 0,74 e 0,81 e a segunda permaneceu entre 0,70 e 0,90. Para avaliar a relação com outras variáveis, as dimensões foram correlacionadas com o Sexual Quality of LifeQuestionnaire(SQ L-M); a satisfação sexual e 0 sofrimento associaram-se moderadamente com 0 instrumento e o controle ejaculatório associou-se levemente com ele. Realizaram-se também comparações entre grupos, e diferenças significativas foram encontradas nas médias entre homens com e sem EP (Althof \& cols., 2006).

Todas essas análises compuseram uma versão preliminar do IPE cujo conteúdo foi avaliado na terceira etapa. Na ocasião, essa versão foi submetida a entrevistas qualitativas com pacientes com queixas de EP. Diferentes modificações foram sugeridas para adequação da linguagem e termos utilizados nos itens. Elas foram incorporadas e constituíram a versão final (Althof \& cols., 2006).
Na quarta e última etapa, uma aplicação da versão final foi realizada. Para avaliação das propriedades psicométricas, utilizou-se a análise fatorial exploratória por componentes principais com rotação promax. A justificativa para mudança do método rotacional foi atribuída às associações encontradas entre as dimensões na segunda etapa. O s critérios para extração foram os mesmos; a análise extraiu três dimensões que explicaram 64,29\% da variância. Mesmo com as modificações de conteúdo feitas na terceira etapa observou-se a manutenção dos itens com as dimensões extraídas anteriormente. No caso, a satisfação sexual reuniu os itens 3,6 , e 8 (cargas fatorias $0,64-0,86$ ), 0 controle ejaculatório permaneceu com os itens $1,2,4$ e 5 (cargas fatorias $0,57-0,80$ ) e 0 sofrimento com itens 9 e 10 (ambos com cargas fatorias 0,93 ). Os coeficientes de precisão por consistência interna e estabilidade temporal apresentaram índices semelhantes aos estimados na segunda etapa (Althof \& cols., 2006).

Para avaliação com outras variáveis, as dimensões foram novamente correlacionadas com o SQL-M e também com o tempo ejaculatório. As associações com SQL-M foram semelhantes, todavia com a dimensão controle mais associada. Com o tempo ejaculatório, elas se associaram de moderado a fortemente. Também foram mencionadas para essas dimensões diferenças de médias entre grupos de homens com e sem EP (Althof $\&$ cols., 2006).

Para conclusão dessas etapas, os autores consideraram a condição multifatorial que compõe 0 fenômeno da EP. Consideraram também o mau uso de um único critério, a exemplo, o tempo ejaculatório, para sua mensuração. Por fim, destacaram o IPE como um instrumento de medida para a avaliação da EP que atende à multidimensionalidade desse fenômeno em conformidade com outros estudos (Byers \& Grenier, 2003; Rowland, 2003).

A sucinta organização exposta sobre a EP apresentou dois desafios para 0 construto. Em primeiro lugar, a necessidade de critérios mensuráveis que possam ser comparados com pesquisas realizadas e futuros estudos. Em segundo, a necessidade de um maior investimento em instrumentos de medida padronizados e validados para avaliar o fenômeno. Althof (2007) acrescenta que essa condição deve ser revertida pelos significativos investimentos realizados pela indústria farmacêutica, na busca de uma alternativa de resolução medicamentosa para aumentar a latência ejaculatória.

Para o Brasil, os desafios são ainda maiores, além de poucos instrumentos de medida que avaliam os aspectos sexuais (Finotelli Jr., 2010), não existem instrumentos que avaliem a EP. Afirma-se que 0 dilema da escassez também é vivido em outros 
construtos e os pesquisadores nessas condições enfrentam 0 impasse relatado por Guillemin, Bombardier e Beaton (1993) em traduzir e adaptar um instrumento ou desenvolver um novo. Segundo esses autores, o processo de tradução e adaptação é uma alternativa interessante, se forem cumpridas algumas diretrizes em relação à compreensão de uma cultura a respeito das diferentes manifestações de uma doença ou dificuldade.

Para contribuir com o cenário exposto, o presente estudo teve como objetivo traduzir e adaptar o Index of Premature Ejaculation (IPE) para o português do Brasil, por método de equivalência semântica. Trata-se de um método amplamente utilizado na literatura, com diretrizes consistentes para comprovar a adequação e adaptação ao contexto cultural da população em questão.

\section{Participantes}

Para 0 processo de avaliação semântica, participaram três profissionais de nível superior em Letras, dois profissionais psicólogos que atuam na área de terapia sexual, um nativo estadunidense, dois profissionais com nível superior em Letras e especialização na língua inglesa, dois psicólogos clínicos com fluência em inglês e um dos pesquisadores deste estudo. Para avaliação da versão criada nesse processo foi solicitado que 50 participantes respondessem à escala e mencionassem sugestões quanto à inteligibilidade dos itens. Ao todo, participaram 34 homens e 16 mulheres, cujas idades variaram entre 18 e 35 anos $(\mathrm{M}=22,12 ; \mathrm{DP}=4,38)$. Todos cursavam uma universidade privada no estado de São Paulo e possuíam nível superior incompleto. A amostra foi composta por conveniência e não foi considerada a possibilidade dos participantes possuírem queixas relacionadas a EP.

\section{Instrumento}

Index of Premature Ejaculation - IPE (Índice de Ejaculação Prematura) (Althof \& cols., 2006). Trata-se de um instrumento em autorrelato, composto por 10 itens em afirmativas que avaliam aspectos da ausência de controle ejaculatório em três dimensões, denominadas satisfação sexual (quatro itens), controle ejaculatório (quatro itens) e sofrimento (dois itens). Os itens são mensurados por escala Liket de cinco pontos com variações entre as questões. Nos itens 1 e 3, a escala varia de cinco pontos para "quase sempre ou sempre" a um ponto para "quase nunca ou nunca"; 0 mesmo para o item 2, em "altamente confiante" a "pouco confiante"; os itens 4 a 7, em "muito satisfeito" a "muito insatisfeito"; 0 item 8, em "muito prazer" a "pouco prazer". E finalmente, para os itens 9 e 10, de um ponto para "extremamente chateado" a cinco pontos para "nada chateado". O escore total e por dimensões é obtido pela soma das respostas dos itens. Com resultado da soma, se padroniza para seus resultados ficarem de 0-100 pontos. Na dimensão 1 e 2 utiliza-se a fórmula (escore bruto - 4) x 100/16, e na dimensão 2, (escore bruto - 2) x 100/ 8. Sua aplicação dura em média 8 minutos, pode ser feita de maneira individual ou coletiva, indicada para homens sexualmente ativos entre 18 e 60 anos. 0 instrumento pode ser aplicado na parceria para verificar a percepção, segundo os aspectos sexuais do parceiro. 0 estudo de criação e validação apresentou evidências de validade baseadas no conteúdo, na estrutura interna e na relação com outras variáveis (Althof \& cols., 2006). Os índices de precisão da escala e dimensões foram estimados pela consistência interna e estabilidade temporal; o primeiro variou de 0,79 a 0,86 e 0 segundo permaneceu entre 0,72 e 0,83.

\section{Procedimento}

Duas autorizações foram obtidas para proceder ao início da pesquisa. A primeira foi fornecida pelos autores do IPE para uso do instrumento; a segunda, pelos responsáveis da universidade, para utilização da amostra.

Em seguida, o projeto foi encaminhado e aprovado pelo Comitê de Etica em Pesquisa (CAAE0429.0.142.000-11). A tradução e adaptação do IPE para o português do Brasil ocorreram por processo de equivalência semântica, conforme sugerido em estudos, a exemplo, Guillemin, Bombardier e Beaton (1993) e Herdman, Fox-Rushby e Badia (1998). As etapas desse processo foram a tradução, a retrotradução, a avaliação da equivalência semântica, a crítica final por especialistas para consideração de outros aspectos e 0 pré-teste da versão.

Para processo de tradução, realizaram-se quatro traduções independentes do instrumento original em inglês para 0 português, duas delas por dois profissionais de nível superior em Letras (tradutor juramentado) e duas por dois profissionais psicólogos que atuam na área de terapia sexual. As quatro versões produzidas foram retrotraduzidas para 0 inglês por um nativo estadunidense e um tradutor juramentado. Ao todo, foram geradas quatro versões do instrumento no português e oito versões no inglês.

As doze versões foram avaliadas por equivalência semântica por dois profissionais com nível superior em letras com especialização em língua inglesa. Nessa avaliação, foram produzidas duas versões independentes (IPE-1 e IPE-2) com a proposta de escolha e incorporação dos melhores termos e frases, 
além de eliminação de discordância. Tratou-se de uma avaliação qualitativa dos itens.

As duas versões criadas, dessa vez, foram avaliadas quantitativamente por juízes independentes, no caso, dois psicólogos clínicos com fluência em inglês (JU1 e JU2). Para cada item do instrumento, os juízes compararam a versão original em inglês do IPE com as duas versões. Nessa comparação, utilizaram uma classificação nominal para averiguar a manutenção do significado de cada item. A escala foi determinada em inalterado (I), pouco alterado (PA), muito alterado (MA) e completamente alterado (CA). Para o resultado dessa avaliação, estimou-se o coeficiente Kappa para indicar o grau de concordância interjuízes.

Após o processo de equivalência semântica, os pesquisadores produziram uma versão preliminar (IPEP) com base nas duas propostas criadas e com resultado quantitativo da avaliação interjuízes. Essa versão foi aplicada coletivamente em uma amostra. $\mathrm{O}$ formulário de aplicação continha um cabeçalho com informações de idade, sexo e escolaridade, a versão preliminar e, ao final, uma pergunta referente a sugestões e/ ou incorporações de termos e/ ou frases não compreendidas.

$\mathrm{Na}$ aplicação, os participantes foram convidados no início da aula e receberam uma breve explicação da pesquisa e seus objetivos. Aqueles que concordaram em participar assinaram as duas vias do Termo de Consentimento Livre Esclarecido e fizeram parte do estudo. Foram fornecidas instruções para responderem à escala e também mencionarem trechos e/ ou palavras nas questões que tiveram dificuldade para entender. Após a aplicação, os pesquisadores consideraram as sugestões e incorporaram aquelas consideradas pertinentes para compreensão do instrumento.

\section{Resultados e discussão}

Após o processo de traduções e retrotraduções, as duas versões criadas por meio da equivalência semântica foram avaliadas. A versão IPE-1 teve os itens 3, 6 e 9 classificados como de significado pouco alterado, segundo a avaliação interjuízes. 0 item 2 apresentou divergência na classificação. 0 restante dos itens foi classificado como de significado inalterado. Já para a versão IPE-2, os itens 4, 7, 8, 10 obtiveram classificações como de significado pouco alterado. O correram divergências nos itens 2 e 3 e os itens restantes foram classificados como de significado inalterado.

O cruzamento entre as classificações do psicólogo JU1 e do psicólogo JU2 apresentou substancial concordância significativa entre os juízes, para ambas as versões (K=0,78-0,80; $\mathrm{p}<0,01)$ (Fonseca, Silva \& Silva, 2007; Landis \& Koch, 1977). Para o IPE-1, 60\% dos itens foram classificados por ambos como inalterados e $40 \%$ como pouco alterados. No IPE-2, $50 \%$ como inalterados e $50 \%$ como pouco alterados. Em nenhuma das versões houve itens classificados como muito alterados e completamente alterados. Esses resultados foram organizados na Tabela 1.

Tabela 1. Frequência das respostas dos juízes segundo classificação proposta para a análise do significado dos itens

\begin{tabular}{|c|c|c|c|c|c|c|c|c|c|c|c|c|c|}
\hline \multirow{2}{*}{\multicolumn{2}{|c|}{ IPE-1 }} & \multicolumn{5}{|c|}{ JU2 } & \multirow{2}{*}{\multicolumn{2}{|c|}{ IPE-2 }} & \multicolumn{5}{|c|}{ JU2 } \\
\hline & & I & PA & MA & CA & Total & & & I & PA & MA & CA & Total \\
\hline JU1 & I & 6 & 1 & - & - & 7 & JU1 & I & 5 & 1 & - & - & 6 \\
\hline & PA & - & 3 & - & - & 3 & & PA & - & 4 & - & - & 4 \\
\hline & MA & - & - & - & - & - & & MA & - & - & - & - & - \\
\hline & CA & - & - & - & - & - & & CA & - & - & - & - & - \\
\hline & Total & 6 & 4 & - & - & 10 & & Total & 5 & 5 & - & - & 10 \\
\hline
\end{tabular}

$(\mathrm{K}=0,78-0,80 ; \mathrm{p}<0,01)$

Segundo esses resultados, o IPE-1 apresentou um grau de concordância menor na avaliação interjuízes em relação ao IPE-2. Por outro lado, ele foi 0 instrumento que recebeu a maior classificação de itens inalterados. Apesar da pequena diferença entre os coeficientes Kappa encontrados na análise, os resultados das discrepâncias sugeriram possibilidades de alterações para composição do IPE-P na avaliação final do especialista.

0 primeiro item analisado nessa avaliação foi a questão 2, que em ambas as versões produziu divergências entre os juízes. Dois pontos foram considerados, sendo o primeiro a diferença entre "0 quão confiante você ficava quando ejaculava" e "0 quão confiante esteve sobre quando ejaculou". Optouse por utilizar "esteve", pois se trata de uma situação passada, e 0 advérbio de intensidade "quão", visto que "confiante" é um adjetivo, sendo "quanto" utilizado para substantivos. Essas alterações constituíram a frase "quão confiante esteve sobre quando ejaculou".

O segundo ponto foi a mudança de "intercurso sexual" ou "penetração sexual e relações sexuais" para "práticas sexuais". Além de termos que remetem a questões técnicas, ambas as expressões se referem à 
prática de penetração especificamente vaginal. A justificativa para essa alteração foi considerar a multiplicidade de atividades sexuais, sendo a queixa da ejaculação precoce encontrada, por exemplo em práticas como carícias, beijos, sexo oral e anal (Jern, 2010; Leiblum, 2007).

D estaca-se que as alterações em relação ao termo "práticas sexuais" e ao uso do "quão" para adjetivos como "confiante", "satisfeitos", "frustrado", entre outros empregados nas duas versões, impactaram alterações na maioria dos itens. Ao se tratar da questão 3 , em que houve divergência no IPE-2 e que foi classificada com significado pouco alterado no IPE-1, foram descartadas as frases "quando teve relações sexuais, com que frequência foi satisfatório para si" e "quando teve penetração sexual, quão frequente era satisfatório para você" para uso direto "quando você teve práticas sexuais, com que frequência elas foram satisfatórias".

Por fim, algumas alterações pontuais foram realizadas nos itens classificados com 0 significado pouco alterado em uma das duas versões. Para a questão 4, foi usado "senso de controle" no lugar de "sentido de controle". Nas questões 6 e 8, foi escolhida a frase proposta no IPE-2, "ficou com sua vida sexual de maneira geral", ao invés de "tem ficado com sua vida sexual no geral", e "as atividades sexuais lhe proporcionaram" no lugar de "em que a relação sexual lhe deu", respectivamente. Outra modificação foi referente à especificação de gênero entre parêntese na questão 7, ao indicar "sua(seu) parceira(o)". Para as questões 9 e 10, foi usado o termo coloquial "chateado" ao invés de "incomodado", terminologia mais conhecida, que dimensiona um grau de maior sofrimento.

D epois de consideradas essas alterações, a versão IPE-P foi construída e aplicada. Poucas foram as sugestões feitas pelos participantes durante a aplicação. A pesar de a maioria achar repetitivo as frases iniciarem com "nas últimas quatro semanas", cinco deles mencionaram que ela ajudou a temporalizar ao fixar 0 período e contexto a ser avaliado. Considera-se que nenhuma alteração foi efetuada, além do acréscimo do termo "(frustrado)" ao lado de "chateado". Acredita-se que esse incremento criou uma dimensão subjetiva do sofrimento na vivência de uma situação em que a ejaculação ocorra rapidamente.

\section{Considerações finais}

É sempre um desafio o processo decisório de uma pesquisa em traduzir e adaptar um instrumento desenvolvido em outra linguagem ou criar um novo. Não se trata somente de prós e contras orientados por importantes referências, mas dos resultados das pesquisas em relação ao construto. No caso, para a EP, observa-se 0 entrave em produzir medidas confiáveis para avaliar o fenômeno. No caso do Brasil, verifica-se a não-existência de instrumentos de medidas para avaliar a EP.

Ainda que o IPE seja considerado como um instrumento adequado para avaliação da EP e mantenha boas qualidades psicométricas, ele necessita de maiores investigações tanto para seu amadurecimento como medida quanto para contribuições das dimensões que propõe avaliar. No caso desta pesquisa, a maior contribuição foi traduzir e adaptar um instrumento de reconhecimento internacional para o português do Brasil, a fim de possibilitar a produção de futuros estudos com a EP.

A tradução por método de equivalência semântica foi a opção escolhida para reduzir a probabilidade de erros nas interpretações das palavras e frases, além de adaptações culturais e da linguagem. Durante esse processo, cada etapa foi detalhada no estudo para garantir a qualidade no processo de tradução e adaptação para o português do Brasil. Considerou-se satisfatório tal processo para o IPE. Para a versão final, conservou-se o nome original em inglês, acrescido ao final de "adaptação brasileira". Esse procedimento foi utilizado também em outros estudos.

Mencionam-se duas limitações para esse processo, a primeira referente à amostra. A conveniência da amostra universitária pode criar um viés na compreensão de algumas questões na comparação com amostra de graus de escolaridade inferiores. Além disso, não houve aplicações em amostra de pacientes com queixas de EP. Essa situação pode implicar na compreensão e comparação de pontuações.

Na segunda, ao invés de um comitê, foi utilizada a participação de dois membros para a crítica final realizada por especialistas no método de equivalência semântica, situação essa ponderada pela qualificação dos profissionais das etapas anteriores e da produção de resultados qualitativos e quantitativos que embasaram os especialistas nas escolhas das palavras e frases que constituíram a versão final do Index of PrematureEjaculation- adaptação brasileira.

Encontra-se em andamento a validação psicométrica dessa versão. Esse processo garantirá, para além da tradução e adaptação, maior confiabilidade ao instrumento para uso por profissionais que necessitem de uma avaliação específica da EP. 0 presente estudo tem um papel importante para a área da psicologia na sexualidade, especificamente nos aspectos clínicos das disfunções sexuais. 


\section{Referências}

Althof, S. (2004). Assessment of rapid ejaculation: review of new and existing measures. Cument Sexual Helth Repats, 1(2), 61-64.

Althof, S. (2007). Treatment of rapid ejaculation. Em S. Leiblum (Ed.), Pinciples and pradice of sex therapy ( $4^{\text {a }}$ ed.). Nova Iorque: The Guilford Press.

Althof, S., Rosen, R., Symonds, T., Mundayat, R., May, K., \& Abraham, L. (2006). Development and validation of a new questionnaire to assess sexual satisfaction, control, and distress associated with premature ejaculation. Joumal of Sexual Mediane, 3(3), 465-475.

Ankier, C., \& Glina, S. (2005). Definições e classificações das disfunções e das respostas sexuais dos homens, ao longo dos tempos. Recuperado: 01 de março de 2005. Disponível: http:/ / www.arquivoshellis.com.br/ revista/ 01_030 205/.

Byers, E. S., \& Grenier, G. (2003). Premature or rapid ejaculation: heterosexual couples'perceptions of men's ejaculatory behavior. Ardives of Sexual Behavior, 32(3), 261-270.

Davies, S., Katz, J., \& Jackson, J. L. (1999). Sexual desire discrepancies: effects on sexual and relationship satisfaction in heterosexual dating couples. Archives of Sexual Behavia, 28(6), 553-567.

Finotelli Jr., I. (2010). Lodking for impowements the brazilian pespeetive on using sexual measuremet instrumets Conferência realizada no X Congress of the European Federation of Sexology, Porto, Portugal.

Fonseca, R., Silva, P., \& Silva, R. (2007). Acordo interjuízes: o caso do coeficiente kappa. Labaratóno dePsicooja, 5(1), 81-90.

Guillemin, F., Bombardier, C., \& Beaton, D. (1993). Cross-cultural adaptation of health-related quality of life measures: Literature review and proposed guidelines. Jamal of Clinical Epidamidogy, 46(12), 1417-1432.

Hartmann, U., Schedlowski, M., \& Kruger, T. H. (2005). Cognitive and partner-related factors in rapid ejaculation: differences between dysfunctional and functional men. Wodd Jamal of Urdog, 23(2), 93-101.

Herdman, M., Fox-Rushby, J., \& Badia, X. (1998). A model of equivalence in the cultural adaptation of HRQ oL instruments: the universalist approach. QualityofLifeRearch, 7(4), 323-335.
Jern, P. (2010). Measuring early ejaculation in same-sex and non-partnered sexual activities. European Urdogical Review 6(1), 46-48.

Kedia, K. (1983). Ejaculation and emission: normal physiology, dysfunction, and therapy. Em R. Krane, M. Siroky \& I. Goldstein (Eds.), Malesexual dysundion(pp. 37-54). Boston: Little, Brown.

Landis, J. R., \& Koch, G. G. (1977). The measurement of observer agreement for categorical data. Biomenics 33(1), 159-174.

Larson, J. H., Anderson, S. M., Holman, T. B., \& Niemann, B. K. (1998). A longitudinal study of the effects of premarital communication, relationship stability, and self-esteem on sexual satisfaction in the first year of marriage. Jaumal of Sex \& Manital Therapy, 24(3), 193-206.

Leiblum, S. R. (2007). Priniples and pradice of sex therapy. Nova Iorque: The Guilford Press.

Levin, R. J. (2005). The mechanisms of human ejaculation: a critical analysis. Sexual andRdationship Therapy, 20(1), 123-131.

Lewis, R. W., Fugl-Meyer, K. S., Bosch, R., FuglMeyer, A. R., Laumann, E. O., Lizza, E., \& Martin-Morales, A. (2004). D efinitions, classification, and epidemiology of sexual dysfunction. Em T. F. Lue, R. Basson, R. C. Rosen, F. Giuliano, S. Khoury \& F. Montorsi (Eds.), Sexual Mediane sexual dysfundions in men and women (pp. 37-72). Paris: Editions 21.

Masters, M. J., \& Johnson, V. E. (1970). Human Sexual Inadequag. Boston: Little, Brown \& Co.

Patrick, D. L., Giuliano, F., Ho, K. F., Gagnon, D. D ., McNulty, P., \& Rothman, M. (2009). The Premature Ejaculation Profile: validation of selfreported outcome measures for research and practice. British Joumal of Urdogy Intemational, 103(3), 358-364.

Porst, H., Vardi, Y., Akkus, E., Melman, A., Park, N. C., Seftel, A. D., Teloken, C., \& Wyllie, M. (2010). Standards for clinical trials in male sexual dysfunctions. Jaumal of Sexual Mediane, 7(1 Pt 2), 414-444.

Rodrigues Jr., O . M. (1995). Psicdoga esexualidade São Paulo: MED SI.

Rowland, D. L. (2003). Treatment of premature ejaculation: selecting outcomes to determine efficacy. Bullein of the Intemational Society for Sexual and Impotence Reserth, 10, 26-27. 
Rowland, D. L., Cooper, S. E., \& Schneider, M. (2001). Defining premature ejaculation for experimental and clinical investigations. Ardhives of Sexual Behavior, 30(3), 235-253.

Rowland, D. L., Strassberg, D. S., de Gouveia Brazao, C. A., \& Slob, A. K. (2000). Ejaculatory latency and control in men with premature ejaculation: an analysis across sexual activities using multiple sources of information. Jaumal of Psychosamatic Reserich, 48(1), 69-77.

Rowland, D. L., Tai, W., \& Brummett, k. (2007) Interactive processes in ejaculatory disorders psychophysiological considerations. Em E. Janssen (Ed.), The Psychphysidogy of Sex (pp. 227243). Bloomington: Indiana University Press.

Rust, J., Golombok, S., \& Collier, J. (1988). Marital problems and sexual dysfunction: how are they related? BitishJaumal of Psychiatry, 152, 629-631.

Segraves, R. T. (2010). Considerations for an evidencebased definition of premature ejaculation in the D SM-V. Jaumal of Sexual Meedione, 7(2 Pt 1), 672679.

Waldinger, M. D. (2007). Premature ejaculation: state of the art. Urdogic Clinics of Noth Ameica, 34(4), 591-599.
Waldinger, M. D ., Hengeveld, M. W., Zwinderman, A. H., \& Olivier, B. (1998). An empirical operationalization study of DSM-IV diagnostic criteria for premature ejaculation. Intemational Jaumal of PsychiatryinClinical Practice, 2(4), 287-293.

Waldinger, M. D., Quinn, P., Dilleen, M., Mundayat, R., Schweitzer, D. H., \& Boolell, M. (2005). A multinational population survey of intravaginal ejaculation latency time. Joumal of Sexual Meeliane, 2(4), 492-497.

Waldinger, M. D., Zwinderman, A. H., Olivier, B., \& Schweitzer, D . H. (2005). Proposal for a definition of lifelong premature ejaculation based on epidemiological stopwatch data. Jaumal of Sexual Meidine, 2(4), 498-507.

Yuan, Y. M., Xin, Z. C., Jiang, H., Guo, Y. J., Liu, W. J., Tian, L., \& Zhu, J. C. (2004). Sexual function of premature ejaculation patients assayed with Chinese Index of Premature Ejaculation. Asian Jamal of Andrology, 6(2), 121-126.

Reedidbem27/ 08/ 2012

Reformuladbem20/ 09/ 2012 Aprovadbem17/ 10/ 2012

Sobre os autores:

Ítor Finotelli Júnior é psicólogo e psicoterapeuta sexual, doutor em Psicologia pelo Programa de Pós-Graduação StridoSensu em Psicologia da Universidade São Francisco.

Cláudio Garcia Capitão é psicólogo, especialista em Psicologia Clínica e Hospitalar, pós-doutorado em Psicologia Clínica pela PUC-SP, docente dos Cursos de Graduação e Pós-Graduação Stricto Sensu em Psicologia da Universidade São Francisco.

Contato com os autores:

Av. Alexandre Rodrigues Barbosa, 45 - CEP 13251-900 - Itatiba/ SP - Brasil.

E-mail: cgcapitao@uol.com.br 